\title{
Time-Resolved Pump-Probe Diffraction Experiment at SOLEIL Synchrotron: Photoinduced Spin Transition in the Molecular Crystal [TPA Fe(III) TCC] $\mathrm{PF}_{6}$
}

\author{
C. Laulhé ${ }^{a} *$, S. Ravy ${ }^{a}$, P. Fertey ${ }^{a}$, E. Elkmaim ${ }^{a}$, F. Legrand $^{a}$, P. Féret $^{a}$, \\ Ph. Hollander ${ }^{a}$, S. Hustache ${ }^{a}$, M. Bordessoule $^{a}$, J.-P. Ricaud ${ }^{a}$, E. Collet $^{b}$,

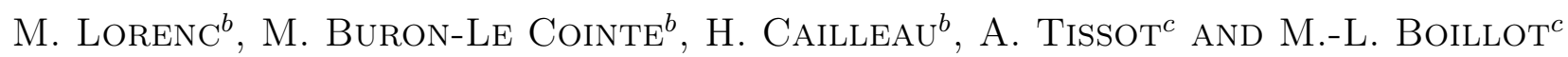 \\ ${ }^{a}$ Synchrotron SOLEIL, L'Orme des Merisiers, Saint Aubin - B.P. 48, 91192 Gif-sur-Yvette, France \\ ${ }^{b}$ Institut de Physique de Rennes, Université de Rennes I - CNRS, UMR 6251, 35042 Rennes, France \\ ${ }^{c}$ Institut de Chimie Moléculaire et Matériaux d'Orsay, UMR-CNRS 8182, Université Paris-Sud 11 \\ 91405 Orsay, France
}

We present the first time-resolved pump-probe experiment performed at synchrotron SOLEIL at the CRISTAL diffraction beamline. The time-resolved setup will be used in the near future for sub-ns time resolution experiments. We studied spin state switching in a [TPA Fe(III) TCC] $\mathrm{PF}_{6}$ single crystal as induced by a $\approx 6 \mathrm{~ns}$ laser pulse $(1064 \mathrm{~nm})$, by measuring the 3D diffraction volume of the 002 Bragg reflection as a function of time after excitation. The intensity profiles (rocking curves) are found to evolve at two characteristic timescales, namely hundreds of ns and few $\mu$ s, exhibiting subtle profile changes and peak broadenings. Consistently with previous studies, we interpret these features as due to structural inhomogeneities related to laser-induced deformation wave propagation and heat diffusion, which both start from the absorbing sites.

PACS: $61.50 . \mathrm{Ks}, 61.05 . \mathrm{cp}, 31.70 . \mathrm{Ks}, 78.47 . \mathrm{J}-$

\section{Introduction}

The SOLEIL synchrotron is currently putting a great effort in developing pump-probe experiments that combine the use of ultrashort laser pulses and the pulsed $\mathrm{X}$-ray beam produced in its storage ring. In such experiments, the time resolution is in practice limited by the X-ray pulse temporal width, which is typically $70 \mathrm{ps}$ FWHM in SOLEIL's temporal operation modes. In view of studying ultrafast photoinduced dynamics in matter, a "low- $\alpha$ " operation mode [1] is currently being tested, which will provide X-ray pulses as short as 1 ps FWHM. An ultimate temporal resolution of $\approx 100 \mathrm{fs}$ is foreseen by 2014 at SOLEIL, in the framework of the "femto-slicing" project [2].

The undulator-based beamline CRISTAL, which is designed for diffraction studies in condensed matter, will use these advanced time structures for pump-probe experiments. In this framework, the sample studied is excited (pumped) by a short laser pulse, and subsequent changes in the sample's atomic structure are studied in the time domain $\Delta t$ by measuring its diffraction out of a monochromatic incident beam. This procedure must be repeated in a stroboscopic way, in order to generate

* corresponding author; e-mail:

claire.laulhe@synchrotron-soleil.fr the necessary statistics and reliability of measurement. At sub-ns and sub-ps resolutions, such experiments allow studying photoinduced phase transitions and out-of-equilibrium phenomena in condensed matter, which bring precious information on photon/matter interaction, as well as on couplings between lattice and other degrees of freedom. On CRISTAL, the sample will be excited by $800 \mathrm{~nm}, 25$ fs FWHM laser pulses provided by a regenerative Ti:sapphire amplifier with tunable repetition rate $[1-10 \mathrm{kHz}]$. The laser will be installed in a dedicated hutch close to the beamline. The $\mathrm{kHz}$ repetition rates are well adapted for pump-probe experiments, because the typical time for dissipating the heat deposited by the laser pulse is about few ms. On the other hand, synchrotron X-ray pulses are generally produced at $\mathrm{MHz}$ frequencies (6.8 MHz in SOLEIL's 8-bunch mode). In order to perform pump-probe experiments, one has to select only one X-ray pulse after each laser excitation, i.e. to separate out X-ray pulses that can be as close as $147 \mathrm{~ns}$ apart. To achieve this goal, we rely on the newly developed 2D detector XPAD3.2 [3]. In this detector, the incoming photons interact with a silicon sensor whose backside is pixelated. Each pixel faces its own electronic chain, which is made of a charge sensitive pre-amplifier devoted to charge detection, a photon energy discriminator, and a counter plus circuitry for the extraction of the data. The sensor and the charge detection stage detect incoming photons continuously, but the counter can 
be enabled or disabled with an external logical signal, or gate. Working frequencies up to $100 \mathrm{kHz}$ and gate durations as short as 90 ns are supported, which gives a great flexibility for choosing the repetition rate and the X-ray pulse selection patterns (70 ps single pulses, or $(N-1) \times 147.8$ ns macro-pulses formed by $N$ single pulses each).

We recently performed the first time-resolved pumpprobe diffraction test experiment on the CRISTAL beamline. The fs laser, which is planned to arrive on October 2011, was not yet available. Instead, we used a Nd:YAG laser which delivers 6 ns FWHM pulses, so that the temporal resolution was limited to the ns range. We considered the molecular crystal [TPA Fe(III) TCC] $\mathrm{PF}_{6}$, where TPA and TCC denote the tris(2-pyridyl-methyl) amine and 3,4,5,6-tetra-chloro-catecholate dianion, respectively. In the monoclinic and orthorhombic polymorphs of this compound, a near infrared laser pulse can switch the spin state of a small portion of Fe atoms in less than $1 \mathrm{ps}$, from a low spin (LS, $S=1 / 2$ ) to a high spin (HS, $S=5 / 2$ ) state $[4,5]$. On the photoexcited molecular sites, the LS to HS transformation is accompanied by an increase of the length of the $\mathrm{Fe}-\mathrm{N}$ bonds which link the $\mathrm{Fe}$ atoms to their ligand TPA [6]. In addition, the phonon population (or lattice temperature) locally increases due to non-radiative decays of electrons from the ligand-to-metal charge transfer states to the HS state. From 10 to few $100 \mathrm{~ns}$ after laser excitation, an increase of the cell parameters $a, b$, and $c$ is observed, which was attributed to the propagation of lattice deformation waves across the solid $[4,7]$. At $\mu$ s delays, one can observe additional activations of molecular switching, following the average temperature increase induced by the laser pulse $[4,7]$. Thermal equilibrium with the environment is finally recovered within $1-10 \mathrm{~ms}$ after laser excitation $[4,8]$. In the following, we report the results of rocking curve profile measurements on the 002 Bragg reflection of orthorhombic [TPA Fe(III) TCC] $\mathrm{PF}_{6}$ (space group $\left.P n a 2_{1}, a \approx 8.4 \AA, b \approx 37.3 \AA, c \approx 9.3 \AA[6]\right)$. Such measurements, which give precious information on lattice plane deformations in samples, are intended to complete our view of the dynamics of the average crystallographic structure described above.

\section{Experimental method}

The sample was oil-fixed on a glass holder. Its temperature was kept at $180 \mathrm{~K}$ during the whole experiment by using an open flow $\mathrm{N}_{2}$ cryostat from Cryo Industries of America. The shape of the crystal is plate-like $\left(175 \times 300 \times 10 \mu \mathrm{m}^{3}\right)$, with the $b$ axis perpendicular to the plate. The sample geometry was arranged such that the X-ray and laser beam incidences are parallel and perpendicular to the sample surface, respectively (Fig. 1).

The sample was excited by $1064 \mathrm{~nm}$ laser pulses at a $10 \mathrm{~Hz}$ repetition rate, with a fluence of $100 \mu \mathrm{J} /\left(\mathrm{mm}^{2}\right.$ pulse $)$. The laser polarization was horizontal and nearly parallel to the crystal axis $a$, which maximizes the penetration depth into the sample [6]. The

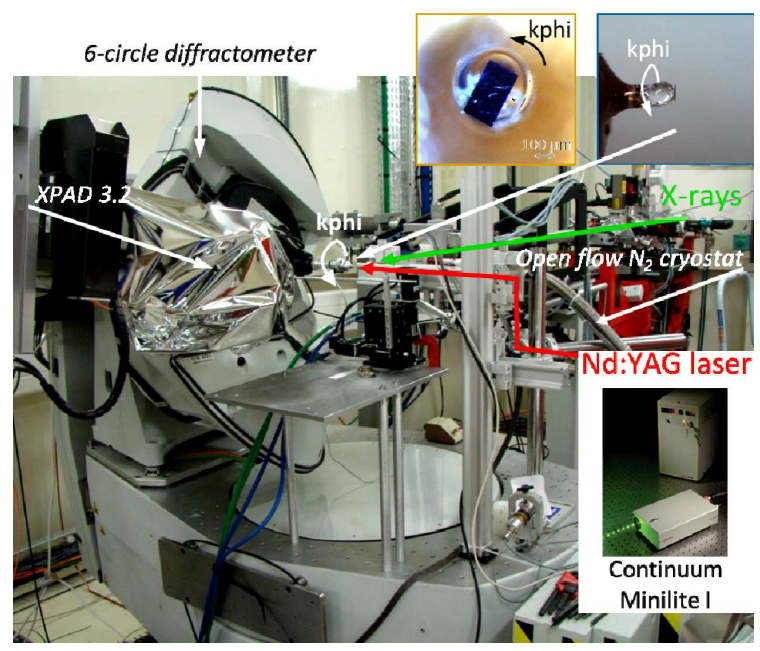

Fig. 1. Experimental setup used for the ns-resolved experiment on CRISTAL beamline. The laser and X-ray beams are represented in (dark) red and (light) green, respectively.

laser spot could be imaged on a paper screen placed at sample position, via backscattering toward a CCD camera. This allowed us to correctly position the laser spot, as well as to estimate its size $(\approx 2 \mathrm{~mm}$ in diameter). SOLEIL's machine was operated in the single-bunch mode, where an X-ray pulse with a temporal width of $70 \mathrm{ps}$ FWHM is produced every $1.18 \mu \mathrm{s}(846.64 \mathrm{kHz})$. The incident X-ray energy was set to $18.623 \mathrm{keV}$. In these conditions, $6 \times 10^{4} \mathrm{ph} /$ pulse reached the sample, with a penetration depth of $1.2 \mathrm{~mm}$ (i.e., the whole sample was probed). The XPAD3.2 detector was set to select and count the diffraction signal from single X-ray pulses at the $10 \mathrm{~Hz}$ working frequency. For delays spanning the range [-400 ns; $200 \mathrm{~ns}$, parasitic counts were observed on the detector's circumference. Though we did not elucidate the exact origin of this signal, we could efficiently reduce the concerned detector's area by wrapping the XPAD with a foil of aluminized mylar (Fig. 1). In order to limit the sample exposure to X-ray radiation, we used a chopper capable to open for about $1 \mathrm{~ms}$ on the $100 \mathrm{~ms}$ period. Timings of the laser, the XPAD3.2 detector and the chopper were all synchronized to the $352.198 \mathrm{MHz}$ radiofrequency signal and phased to the $846.64 \mathrm{kHz}$ storage ring clock, via a FPGA technology.

The rocking curves were measured by rotating the sample about the laser beam axis ("kphi" angle as shown in Fig. 1). The counting time was $20 \mathrm{~s}$ per $0.025^{\circ}$ step. The distance between the XPAD3.2 detector and the sample was of $497 \mathrm{~mm}$.

\section{Results}

The 002 reflection images can first be summed over the kphi-angle, in order to get the contour of the whole diffracted intensity. Figure 2 shows the temporal evolution of the summed images. One can observe that the 002 
diffraction peak undergoes significant broadening $300 \mathrm{~ns}$ after laser excitation, narrows back, and then broadens again at $\mu$ s delays before recovering its original shape.
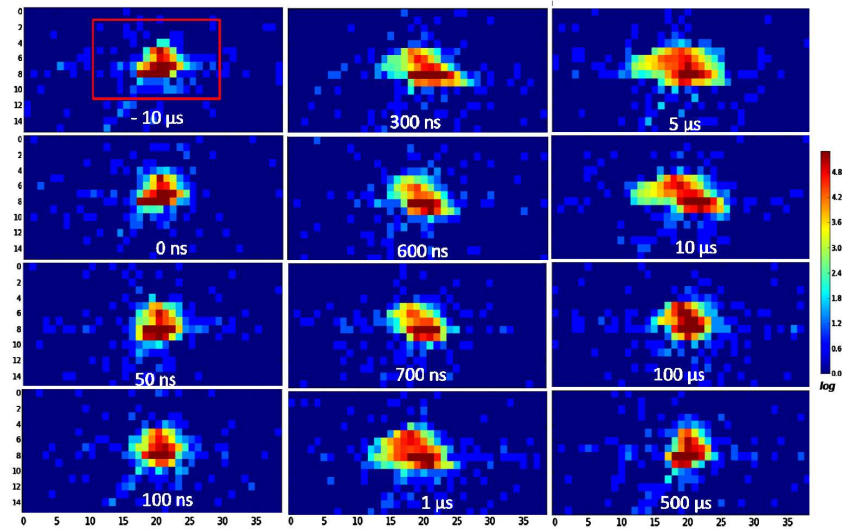

Fig. 2. 002 Bragg spot in orthorhombic [TPA Fe(III) TCC] $\mathrm{PF}_{6}$, integrated during a $0.35^{\circ}$ kphi-oscillation, for various delays after laser excitation (counting time: $20 \mathrm{~s}$ per $0.025^{\circ}$ steps in kphi). The delay is indicated in each part. The rectangular zone shown in the top left part was used to calculate the rocking curves presented in Fig. 3. Each pixel corresponds to $0.015^{\circ}$.

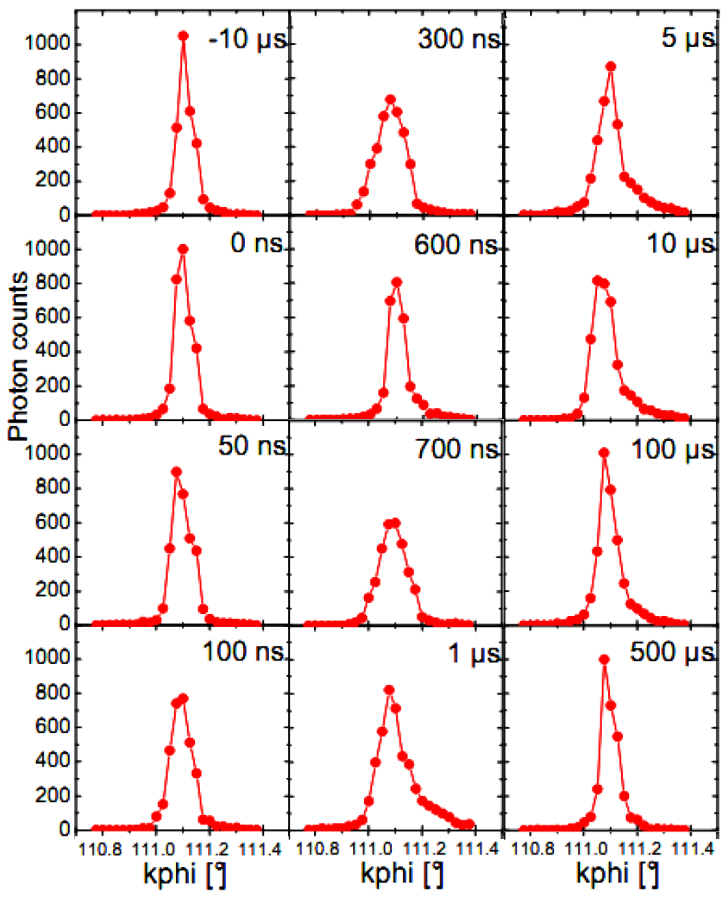

Fig. 3. Rocking curve intensity profile of the 002 Bragg reflection in orthorhombic [TPA Fe(III) TCC] $\mathrm{PF}_{6}$, for various delays after laser excitation.

In order to obtain a more quantitative description of the diffraction profiles, we now consider the rocking curves of the 002 reflection (Fig. 3). In this figure, each data point is the sum of pixel intensities in the detector region indicated on top left part of Fig. 2, so that the width of the rocking curves simply corresponds to the width of 002 reflection in the direction perpendicular to the images presented in Fig. 2 (i.e., approximately the $a^{*}$ direction of reciprocal space). The rocking curve profiles exhibit a significant broadening in the [100 ns-100 $\mu \mathrm{s}$ ] delay-range, in which two typical timescales can further be distinguished:

(1) At hundreds of ns delays, the rocking curve profile hardly changes and undergoes simple broadening.

(2) At $\mu$ s and tens of $\mu$ s delays, a large tail appears on the high kphi-angle side of the rocking curves.

We report the time-dependence of the full widths at half maximum (FWHM) and at $1 / 20$ of maximum in Fig. 4a,b, respectively. The FWHM exhibits two maxima at $200 \mathrm{~ns}$ and $1 \mu \mathrm{s}$, both having roughly the same amplitude. The width at $1 / 20$ of maximum evolves with the same characteristic timescales as the FWHM. Nevertheless, it is twice smaller at $200 \mathrm{~ns}$ than at $1 \mu \mathrm{s}$.

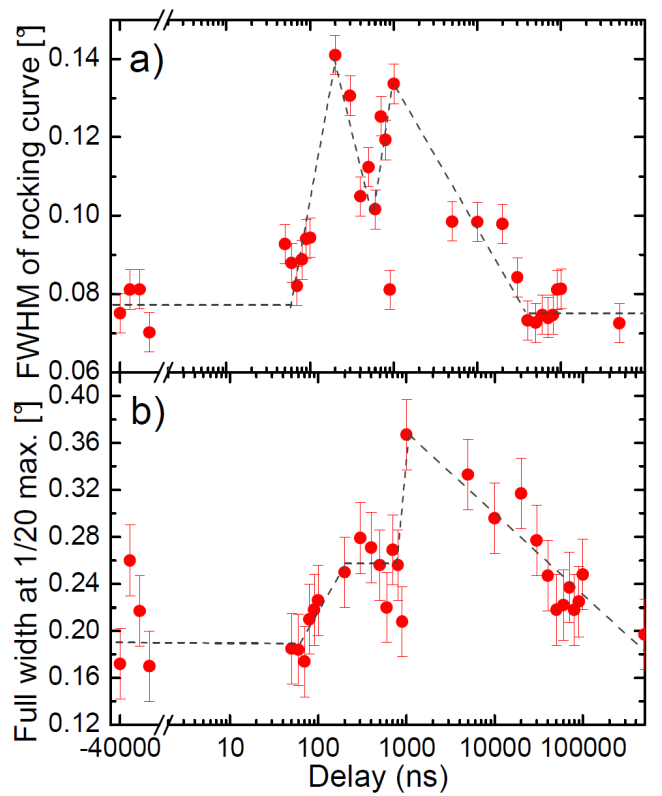

Fig. 4. Time-dependence of (a) full width at half maximum (FWHM) and (b) full width at $1 / 20$ of maximum of the rocking curve intensity profiles of [TPA Fe(III) $\mathrm{TCC}] \mathrm{PF}_{6}$.

Diffraction peak broadening is usually interpreted as an increase in crystal mosaicity. In this respect, our observations give evidence for significant deformations of the (002) lattice planes at two characteristic delays after laser excitation: $\approx 200 \mathrm{~ns}$ and $\approx 1 \mu \mathrm{s}$. The rocking curve profiles strongly differ at these two delays, indicating that the deformations which occur $200 \mathrm{~ns}$ and $1 \mu$ s after laser excitation are of different nature. Furthermore, the characteristic timescales evidenced for the lattice plane distortions fairly correspond to the times of deformation wave propagation (few hundreds of ns after laser excitation) and thermal activation of molecular switching (few 
$\mu$ s after laser excitation) that were observed in previous time-resolved structural studies [4, 7]. On the basis of these observations, we conclude that [TPA Fe(III) TCC] $\mathrm{PF}_{6}$ crystals undergo two successive steps of strong lattice plane deformations, which could be related to the transient and inhomogeneous structures that result from consecutive propagation of deformation waves, and thermal spin-state switching.

\section{Conclusion}

A time-dependent diffraction signal was observed for the first time on the CRISTAL beamline at the ns temporal resolution. This experiment allows us to validate the methodology and technical setups employed, which should also give access to 70 ps time-resolution by using an fs-laser as the pump.

We give evidence for two cycles of strong lattice plane deformations in [TPA Fe(III) TCC] $\mathrm{PF}_{6}$ crystals, which likely result from consecutive laser-induced strain and laser heating. The full analysis of the peak-shape time-dependence will be presented in a forthcoming publication.

\section{Acknowledgments}

This work was supported by the ANR (BLAN-0212 project).

\section{References}

[1] J. Feikes, K. Holldack, P. Kuske, G. Wüstefeld, in: Proc. EPAC'04 - WEPLT session, Lucerne 2004 , p. 1954.

[2] A. Nadji, F. Briquez, M.-E. Couprie, J.-C. Denard, J.-M. Fihlol, Ph. Hollander, C. Herbaux, M. Labat, J.-F. Lamarre, C. Laulhé, V. Leroux, J. Lüning, O. Marcouillé, J.-L. Marlats, M. Meyer, T. Moreno, P. Morin, P. Prigent, S. Ravy, F. Sirotti, in: Proc. IPAC'10 - WEPEA session, 2010, p. 2499.

[3] S. Hustache, K. Medjoubi, J.-C. Clemens, C. Laulhé, $\mathrm{S}$. Ravy, in preparation.

[4] M. Lorenc, J. Hébert, N. Moisan, E. Trzop, M. Servol, M. Buron-Le Cointe, H. Cailleau, M.-L. Boillot, E. Pontecorvo, M. Wulff, S. Koshihara, E. Collet, Phys. Rev. Lett. 103, 028301 (2009).

[5] N. Moisan, M. Servol, M. Lorenc, A. Tissot, M.-L. Boillot, H. Cailleau, S. Koshihara, E. Collet, C.R. Chimie 11, 1235 (2008).

[6] E. Collet, M.-L. Boillot, J. Hebert, N. Moisan, M. Servol, M. Lorenc, L. Toupet, M. Buron-Le Cointe, A. Tissot, J. Saintonb, Acta Crystallogr. B 65, 474 (2009).

[7] E. Collet, M. Lorenc, M. Cammarata, L. Guérin, M. Servol, A. Tissot, M.L. Boillot, H. Cailleau, M. Buron-Le Cointe, Chemistry - A European Journal, doi: $10.1002 /$ chem.201103048.

[8] C. Enaschescu, A. Hauser, Jean-Jacques Girerd, Marie-Laure Boillot, Chem. Phys. Chem. 7, 1127 (2006). 